Anaesthesist 2013 $\cdot 62: 517-518$

DOI 10.1007/s00101-013-2200-z

Online publiziert: 30. Juni 2013

c) Springer-Verlag Berlin Heidelberg 2013

\section{J. Meier}

Klinik für Anästhesiologie und Intensivmedizin, Tübingen

„Blut ist dicker als Wasser"
Im Jahr 1930 wurde dem Österreicher Karl Landsteiner der Nobelpreis für Physiologie und Medizin für seine Entdeckung des AB0-Systems der Blutgruppen verliehen. Er hatte 1901 erkannt, dass eine Bluttransfusion zwischen Personen der gleichen Blutgruppe nicht zur Zerstörung der Blutzellen führte, wohl aber die zwischen Personen verschiedener Blutgruppen. Auf seinen Arbeiten basierend konnte 1907 die erste erfolgreiche Bluttransfusion am Mount Sinai Hospital in New York von Reuben Ottenberg durchgeführt werden [1].

Etwas mehr als 100 Jahre später gehört die Transfusion von allogenen Blutprodukten zum Standardrepertoire der perioperativen Medizin. Viele große Eingriffe wären ohne die Möglichkeit, das verlorene Blutvolumen durch die Gabe von Fremdblut zu ersetzen, undenkbar. Der Fortschritt der operativen Medizin war aus diesem Grund für viele Jahre von der Bereitstellung von Fremdblutkonserven und damit dem Altruismus einer zunehmenden Zahl von Blutspendern abhängig. Die intraoperative Gabe von Fremdblut wurde in der Bevölkerung als elementarer Bestandteil der modernen Chirurgie gesehen, und jede Blutspende half, den Fortschritt zu sichern und „Leben zu retten“.

\ Die „Aids-Krise“ änderte vieles.

Mit Auftreten der Immunschwächekrankheit Aids in den 1980er Jahren und dem damit entstehenden Bewusstsein der Bevölkerung, dass die Transfusion von Fremdblut mit einem relevanten Infektionsrisiko verbunden sein kann, änderte sich diese Einstellung schlagartig. Zusätzlich rückten weitere Nebenwirkungen, wie z. B. transfusionsassoziierte Verschlechte- rungen der Lungenfunktion immer mehr ins Zentrum des wissenschaftlichen Interesses [2]. Obwohl heutzutage moderne Screeningverfahren die Gefahr einer viralen Infektion über Blut oder Blutprodukte auf ein sehr niedriges Niveau reduziert haben, gehört die potenzielle Infektionsgefahr nach wie vor zu den größten Ängsten, die in diesem Zusammenhang aufseiten der Patienten existieren [3].

Die Wahrheit liegt aber, wie so oft, in der Mitte: Weder ist die Gabe von Fremdblut in jeder Situation lebensrettend, noch ist sie mit unkalkulierbaren Risiken verbunden. Blut sollte vielmehr als Medikament mit klarer Indikation und relevantem Nebenwirkungsspektrum gesehen werden. Im Fall nebenwirkungsärmerer Alternativen sollte aus diesem Grund auf die Gabe von Blut komplett verzichtet werden. Allerdings war bis vor wenigen Jahren nicht unbedingt klar, welche Bedeutung die einzelnen Alternativen zur Fremdbluttransfusion besitzen. In vielen bisherigen Untersuchungen wurde in diesem Zusammenhang eine Entität (z. B. Anwendung der maschinellen Autotransfusion, Eigenblutspende, akute normovolämische Hämodilution u. a.) untersucht, und im Anschluss der Effekt auf die Notwendigkeit zur Transfusion von Fremdblut beschrieben [4]. Erst seit Kurzem wächst das Bewusstsein, dass nicht die Verhinderung einer Transfusion durch die eine oder andere singuläre Maßnahme zwingend zu einer Verbesserung der Situation des einzelnen Patienten führen muss, sondern dass vielmehr der patientenorientierte Einsatz eines mehrstufigen Maßnahmenbündels zu Prävention und Therapie einer perioperativen Anämie höchst effektiv Morbidität und Letalität reduzieren hilft [5]. Dieses „patient 
blood management" (PBM) ist ein multidisziplinäres, evidenzbasiertes, mehrstufiges Behandlungsmodell, bei dem der perioperative Einsatz von Blut und Blutprodukten durch folgende Maßnahmen optimiert werden kann [6]:

- Optimierung des Erythrozytenvolumens,

- Minimierung von Blutungen und Blutverlusten sowie

- Erhöhung und Ausschöpfung der natürlicherweise vorhandenen Anämietoleranz.

> Der ganzheitliche, patientenorientierte Ansatz ist der Schlüssel.

Hierbei geht es nicht darum, möglichst niedrige Hämoglobinwerte zu tolerieren, um die Gabe von Fremdblut um jeden Preis zu verhindern. Vielmehr ist es das Ziel, durch den intelligenten Einsatz aller zur Verfügung stehenden Möglichkeiten die potenzielle Transfusionsbedürftigkeit eines Patienten gar nicht erst entstehen zu lassen.

Überraschenderweise ist keine der einzelnen propagierten Maßnahmen revolutionär: Nahezu jede der einzelnen Empfehlungen wird bereits seit vielen Jahren im klinischen Alltag umgesetzt. Innovativ am PBM ist vielmehr der ganzheitliche, patientenorientierte Ansatz, weg von einem Blutprodukt, z. B. dem Erythrozytenkonzentrat, hin zu einem patientenorientierten, optimierten Behandlungspfad.

Trotz des vielversprechenden Konzepts erfordert die individuelle Initiierung eines PBM an einer Klinik Durchhaltevermögen und Überzeugungsarbeit. Patient blood management bedeutet eben nicht nur, auf die eine oder andere Transfusion im Rahmen einer Operation zu verzichten, sondern verpflichtet den behandelnden Arzt, während des gesamten Krankenhausaufenthalts des Patienten dessen Anämie nicht als Labordeviation zu verstehen, sondern als therapiebedürftige Erkrankung, die perioperativ quoad vitam eine entscheidende Rolle spielt.

Ähnlich verfahren wir bereits mit kardiovaskulären Erkrankungen. Niemand käme auf die Idee, einen Patienten mit kardiovaskulärer Risikokonstella- tion einem großen Eingriff ohne vorherige Abklärung und Therapieoptimierung zu unterziehen; dagegen wird eine akute Anämie, die ein ähnliches perioperatives Risiko besitzt, regelhaft ignoriert. Dies ist umso bedenklicher, da sich dieses Risiko in den meisten Fällen eben nicht zwangsläufig durch die Transfusion von Erythrozyten verringern lässt [7].

Mehr als 100 Jahre nach der Entdeckung der Blutgruppen ist es wieder eine österreichische Arbeitsgruppe, die es in Angriff genommen hat, den Umgang mit Blut für jeden Patienten individuell sicherer zu machen. Gombotz u. Hofmann beschreiben in ihrem Beitrag „Patient blood management" zur vorliegenden Ausgabe von Der Anaesthesist leichtverständlich die oben genannten 3 Säulen, die helfen, einen Patienten optimal während eines operativen Eingriffs zu begleiten. Für den Leser bleibt allerdings die Aufgabe, all die wertvollen Ratschläge im eigenen klinischen Umfeld in die Tat umzusetzen.

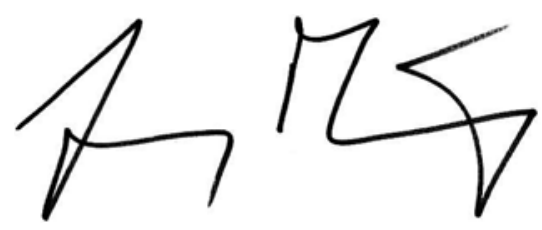

J. Meier

\section{Korrespondenzadresse}

Prof. Dr. J. Meier

Klinik für Anästhesiologie und Intensivmedizin Hoppe-Seyler-Str. 3, 72076 Tübingen

jens.meier@med.uni-tuebingen.de

Interessenkonflikt. Der korrespondierende Autor gibt an, dass kein Interessenkonflikt besteht.

\section{Literatur}

1. Heidelberger M, Landsteiner K (1923) On the antigenic properties of hemoglobin. J Exp Med 38:561-571

2. Tank S, Sputtek A, Kiefmann R (2013) Transfusionsassoziierte akute Lungeninsuffizienz. Anaesthesist 62:254-260

3. Lee $D$ (2006) Perception of blood transfusion risk. Transfus Med Rev 20:141-148

4. Hansen E, Seyfried T (2011) Maschinelle Autotransfusion. Anaesthesist 60:381-389

5. Moskowitz DM, McCullough JN, Shander A et al (2010) The impact of blood conservation on outcomes in cardiac surgery: is it safe and effective? Ann Thorac Surg 90:451-458
6. Goodnough LT, Shander A (2012) Patient blood management. Anesthesiology 116:1367-1376

7. Musallam KM, Tamim HM, Richards T et al (2011) Preoperative anaemia and postoperative outcomes in non-cardiac surgery: a retrospective cohort study. Lancet 378:1396-1407 